REVISTA DE METALURGIA, 48 (1)

ENERO-FEBRERO, 13-23, 2012

ISSN: $0034-8570$

elSSN: $1988-4222$

doi: 10.3989/revmetalm.1109

\title{
Taladrado por fricción en aceros de doble fase $e^{(\cdot)}$
}

\author{
D. Nardi*, L. N. López de Lacalle* y A. Lamikiz*
}

Resumen

\begin{abstract}
En este trabajo se expone un estudio del proceso de taladrado por fricción en chapas de diferentes aceros de doble fase (DP), con límites elásticos variables desde 600 hasta $800 \mathrm{MPa}$. Se realiza una evaluación de las condiciones óptimas de mecanizado en función del material y el espesor empleado, variando las velocidades de giro y los avances de penetración. Al mismo tiempo se monitoriza el par y la fuerza axial resultante, analizando además el espesor de la rebaba en distintas profundidades, es decir, las tolerancias dimensionales generadas por el proceso. Otro punto de interés es la medición de la temperatura alcanzada durante el proceso como consecuencia de la fricción, ya que la cantidad de calor absorbido por la pieza puede influir en las transformaciones microestructurales que el material experimenta. Esta afectación térmica puede transformar las propiedades mecánicas del material en las cercanías del agujero y en la rebaba.
\end{abstract}

Palabras clave Taladrado por fricción; Aceros de alta resistencia; Aceros doble fase.

\section{Friction drilling of dual phase steels}

\begin{abstract}
This work describes the experimental study of the friction drilling process in dual phase (DP) steels, with yield strengths from 600 to $800 \mathrm{Mpa}$. The optimal machining conditions with different thicknesses was assessed through controlled tests at different rotation speeds and feed rates. On one hand, the torque and the thrust force were computed and monitorized. On the other hand, the dimensional tolerances of the holes were evaluated. Another topic of interest inherent to this special technique is the temperature level reached during the friction process which is crucial when it comes to development of microstructural transformations. This thermal condition can transform the mechanical properties of material near the hole and the burr.
\end{abstract}

Keywords

Friction drilling; High strength steels; Dual phase steels.

\section{INTRODUCCIÓN}

El taladrado por fricción es un proceso 'no convencional' de generación de agujeros en chapas. Una de las características de este proceso es que la herramienta rotativa carece de filos de corte, estando su geometría definida básicamente por dos secciones distintas: una superficie cónica y otra cilíndrica. El proceso emplea el calor generado por la fricción entre la parte cónica de la herramienta y la pieza, lo que provoca un ablandamiento del material permitiendo penetrar en él y generando a su vez una rebaba en la salida del agujero ${ }^{[1-3]}$. La parte cilíndrica es la encargada de definir el diámetro final del agujero.

El proceso presenta muchas ventajas frente a procesos tradicionales, pues en un proceso de tala- drado tradicional se hace necesario el empleo de refrigeración con el fin de reducir la fricción y el calor generado, justo lo contrario de lo que pretende esta técnica. Por lo tanto al no aplicar refrigeración se convierte en un proceso limpio. Además, la rebaba generada es susceptible de ser roscada, lo que permite sustituir el empleo de tuercas soldadas. Esta aplicación cobra gran interés cuando se trata de hacer agujeros roscados en piezas tubulares o con geometrías complejas que no permitan un fácil acceso. La mayor parte del material de la pieza en contacto con la herramienta pasa a formar parte de la rebaba generada (Fig. 1) en la parte inferior de la pieza y otra pequeña parte del material genera rebaba en la parte superior. Existen dos opciones cuando se trata de la rebaba superior, una posibilidad es que sea aplastada por el anillo de la herramienta

(·) Trabajo recibido el 02 de febrero de 2011 y aceptado en su forma final el día 01 de julio de 2011.

* Departamento de Ingeniería Mecánica, Universidad del País Vasco, c/ Alameda Urquijo, s/n, 48013, Bilbao, España. 
y ese material permanezca en la pieza, lo que implica que es un proceso sin generación de viruta. La otra posibilidad es que ese material sea eliminado de la pieza; para ello la herramienta está dotada de un rompevirutas entre el anillo y la zona cilíndrica encargada de definir el diámetro del agujero. Pero como la cantidad de material eliminado es escasa sigue siendo un proceso limpio sin generación de viruta. Por otra parte la vida útil de la herramienta es prolongada, ya que carece de filos de corte que se desgasten.

Esta investigación se lleva a cabo en aceros de fase doble (aceros dual phase o DP) de gran uso en la industria del automóvil, ya que poseen simultáneamente unas altas características mecánicas junto con una buena conformabilidad y soldabilidad. Son aceros de bajo contenido en carbono, muy poco aleados, que requieren un tratamiento intercrítico en el dominio austenita+ferrita, seguido de un enfriamiento lo suficientemente rápido como para transformar la austenita en martensita. Este tratamiento se aplica normalmente en línea, utilizando instalaciones de recocido en continuo de chapas. Las propiedades finales de estos aceros dependen de la fracción volumétrica de sus dos fases constitutivas, ferrita y martensita, que a su vez dependen de su contenido en carbono, de la temperatura del tratamiento intercrítico y de la facilidad de temple, que a su vez es función del contenido en elementos de aleación y del espesor de la chapa. En este trabajo se han tomado una serie de aceros DP comerciales y se empleó el ensayo de taladrado por fricción para realizar su caracterización, llegando a obtener los valores ideales del proceso.

\section{MATERIALES Y PROCEDIMIENTO EXPERIMENTAL}

Los ensayos se llevaron a cabo en un centro de mecanizado vertical de tres ejes, gobernados por control numérico, $\operatorname{Kondia}^{\odot}$ modelo K76, siendo capaz de alcanzar $4000 \mathrm{rpm}$. Fue necesario el diseño de un utillaje para la sujeción de las probetas durante los ensayos. Este utillaje permitió alinear la probeta con los ejes de la máquina y evitar la deformación transversal de la pieza durante la operación de taladrado por fricción.

Se diseñó una campaña de ensayos en la que se reflejaron los principales parámetros que afectan al proceso, i) espesor del material, ii) velocidad de giro y iii) velocidad de penetración de la herramienta. Se experimentó con dos espesores y composiciones químicas distintas como se muestra en la tabla I. Para cada uno de los espesores se seleccionaron cuatro velocidades de giro del husillo de la máquina, y para cada velocidad de rotación se ensayaron distintos avances de penetración de la herramienta. Las condiciones del proceso empleadas en la ejecución de los ensayos están reflejadas en la tabla II.

Las herramientas empleadas son de carburo de tungsteno con matriz de cobalto, de la marca Formdrill $^{\circ}$. La geometría de la herramienta de tala-

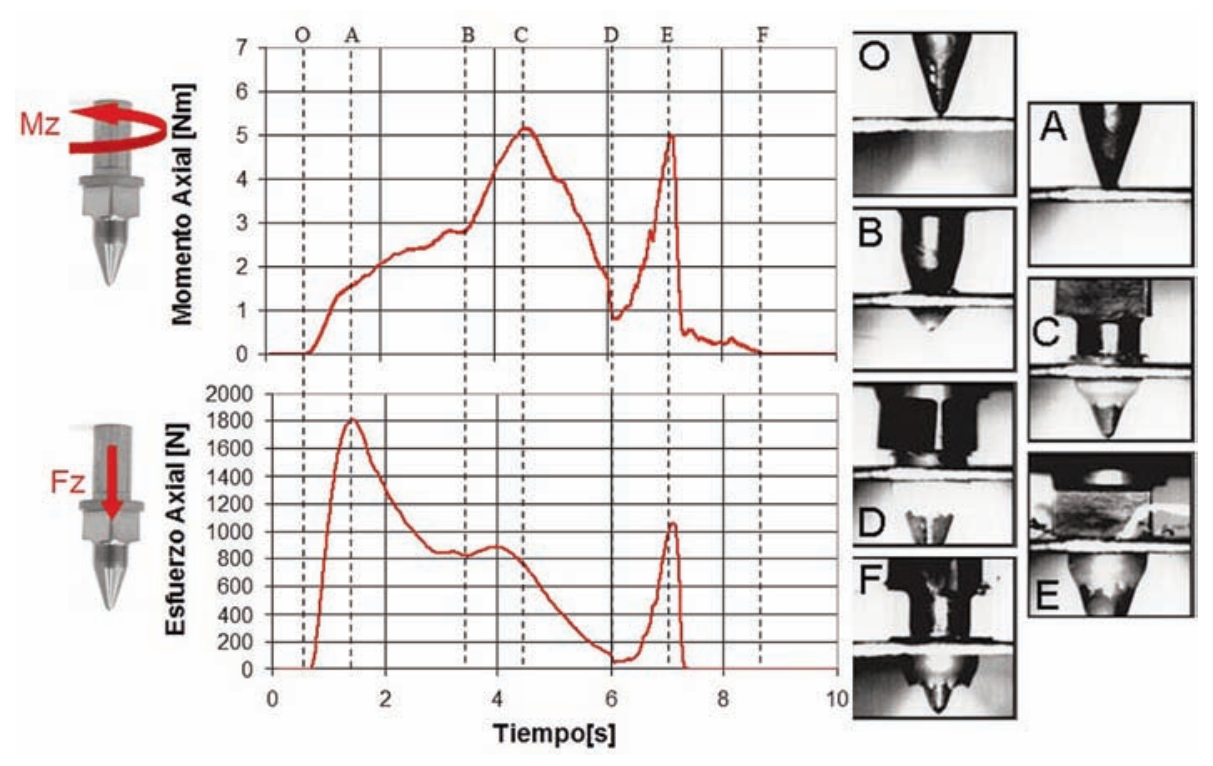

Figura 1. Etapas del proceso de taladrado por fricción.

Figure 1. Illustration of stages in the friction drilling process. 
Tabla I. Composición química de los aceros de Doble Fase

Table I. Chemical composition of Dual Phase steels

\begin{tabular}{lccccccc}
\hline & C & Si & Mn & P & S & Cr & Al \\
\hline DP 600 & 0,09 & 0,25 & 1,93 & 0,02 & 0,005 & 0,21 & 0,034 \\
DP 800 & 0,16 & 0,25 & 1,9 & 0,02 & 0,005 & 0,5 & 0,015 \\
\hline
\end{tabular}

Tabla II. Condiciones del proceso de taladrado por fricción empleadas en los ensayos

Table II. Friction drilling parameters

\begin{tabular}{|c|c|}
\hline Parámetros & \\
\hline Espesor de la chapa (mm) & 1,$2 ; 1,6$ \\
\hline Velocidad de giro (rpm) & $1500,2000,2500,3000$ \\
\hline $\begin{array}{l}\text { Velocidad de Avance } \\
\text { (mm/min) }\end{array}$ & $\begin{array}{l}130,140,170,180,210,220,230,240,270, \\
280,310,320,330,340,370,380,410,420\end{array}$ \\
\hline $\begin{array}{l}\text { Diámetro de la herramienta } \\
(\mathrm{mm})\end{array}$ & 7,3 \\
\hline
\end{tabular}

drado está dividida en 6 secciones, como se puede apreciar en la figura 2.

La herramienta empleada Formdrill ${ }^{\circ}$ presenta como medidas: $\varnothing_{c}=7,3 \mathrm{~mm}, \varnothing_{d}=8 \mathrm{~mm}, \beta=40^{\circ}$, $\alpha=90^{\circ}$, zona de centrado $h_{c}=0,9 \mathrm{~mm}$, zona cónica $h_{i}=7,6 \mathrm{~mm}$, zona cilíndrica $h_{l}=5,4 \mathrm{~mm}$ y la zona del mango $h_{\mathrm{s}}=15 \mathrm{~mm}$.

Dos variantes del Dual Phase fueron probados en este ensayo y con dos espesores distintos: el DP 600 con $1,2 \mathrm{~mm}$ de espesor y el DP 800 con $1,6 \mathrm{~mm}$ de

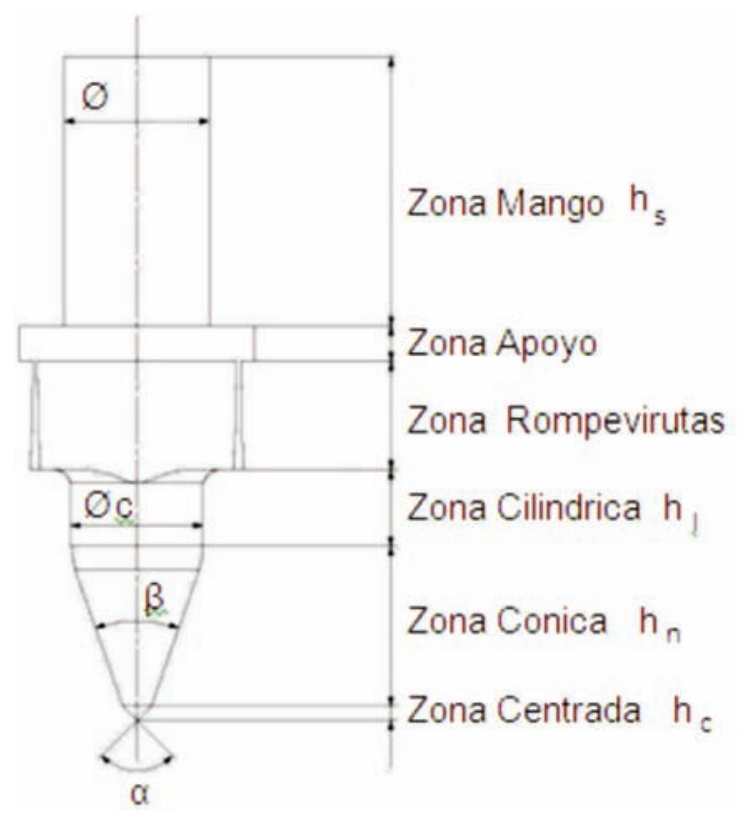

\begin{tabular}{|cc|}
\hline$h_{\mathrm{s}}$ & $15 \mathrm{~mm}$ \\
\hline$\varnothing$ & $8 \mathrm{~mm}$ \\
\hline $\mathrm{h}_{1}$ & $5.4 \mathrm{~mm}$ \\
\hline$\varnothing_{\mathrm{c}}$ & $7.3 \mathrm{~mm}$ \\
\hline $\mathrm{h}_{\mathrm{n}}$ & $7.6 \mathrm{~mm}$ \\
\hline $\mathrm{h}_{\mathrm{c}}$ & $0.9 \mathrm{~mm}$ \\
\hline$\beta$ & $40^{\circ}$ \\
\hline$\alpha$ & $90^{\circ}$ \\
\hline
\end{tabular}

Figura 2. Geometría de la herramienta.

Figure 2. Design of the friction drill tool. 
espesor. La principal característica de este tipo de aceros es el incremento del límite elástico tras dos procesos principales. Uno de ellos es una deformación superior al $2 \%$, valor que se incrementa entorno a $100 \mathrm{Mpa}$ en calidades como el DP 600. El otro proceso que incrementa el límite elástico es un calentamiento a $170^{\circ} \mathrm{C}$ durante 20 min denominado como 'bake-hardening'. Además, estos aceros conservan intactas sus propiedades mecánicas hasta $\operatorname{los} 300^{\circ} \mathrm{C}$, e incluso para temperaturas superiores disminuyen el límite elástico y resistencia de manera gradual y no brusca.

La caracterización de las fuerzas del proceso-herramienta-material es de gran utilidad, ya que permiten entender la interacción del proceso de fricción y el posterior corte de la rebaba superior. Se han realizado estudios con el fin de realizar modelos de la fuerza de empuje y momento axial, así como para calcular los coeficientes de fricción y los esfuerzos de cortadura $^{[5}$ y 6 . En la medición de la fuerza de empuje y momento se empleó una mesa dinamométrica Kistler $^{\circledR}$, modelo 9255B. Para la medición del par se deben considerar las contribuciones de las fuerzas en las direcciones $X$ e $Y$, tanto en módulo como en dirección, en cada uno de los piezoeléctricos y multiplicarlas por la distancia al centro de la mesa. Las señales de los piezoeléctricos son sumadas analógicamente y amplificadas a un voltaje proporcional. Estos voltajes son acondicionados, visualizados y grabados en tiempo real mediante el analizador $\mathrm{LMS}^{\mathbb{\odot}}$ Scada Mobile con el uso del un equipo de cómputo.

La temperatura alcanzada por la pieza es un factor importante en la formación de la rebaba. Cuando la temperatura alcanzada durante el taladrado por fricción es baja, la viruta experimenta un mayor número de fracturas, desplazando el material en dirección radial al agujero. Por otro lado, cuando la temperatura alcanzada por la pieza es mayor, la rebaba adquiere una forma más cilíndrica ${ }^{[3 \text { y } 4]}$. Las temperaturas generadas en el proceso de taladrado fueron recogidas mediante el empleo de un pirómetro de dos colores, modelo Impac ${ }^{\circ}$ IGAR $12 \mathrm{LO}$, el cual puede medir temperaturas comprendidas en el rango de $350^{\circ} \mathrm{C}$ hasta $1.300^{\circ} \mathrm{C}$. La captura de la señal se realizó en tiempo real con la ayuda de un ordenador y el software específico para la marca y modelo del pirómetro empleado.

Durante el proceso, el material es sometido simultáneamente a diferentes tasas de deformación y temperatura, cuya zona de fluencia sigue hasta la zona adyacente al agujero. Debido a la presencia de estos gradientes de deformación y temperatura en la zona termomecánicamente afectada (ZAC) del agujero final, este se puede ocurrir de forma simultánea con diferentes cambios microestruturales. Para el análisis de este fenómeno, fue utilizado como herramienta un durómetro de microdureza Vickers, del fabricante Future-Tech, modelo FV-700e, con una precarga de $490,3 \mathrm{~N}$.

El diámetro del agujero, la profundidad total de la rebaba y los espesores de ésta, se ven afectados por la elección de los parámetros de taladrado. Se realizó un corte transversal de los agujeros, de manera que la medición de las características anteriormente mencionadas se pudo hacer de manera sistemática y precisa.

Para caracterizar el espesor de la rebaba se realizaron mediciones a determinadas profundidades, como se puede observar en la figura 3. Se empleó un microscopio de medida, Mitutoyo ${ }^{(} \mathrm{TM}-100$, que dispone de una óptica de treinta aumentos.

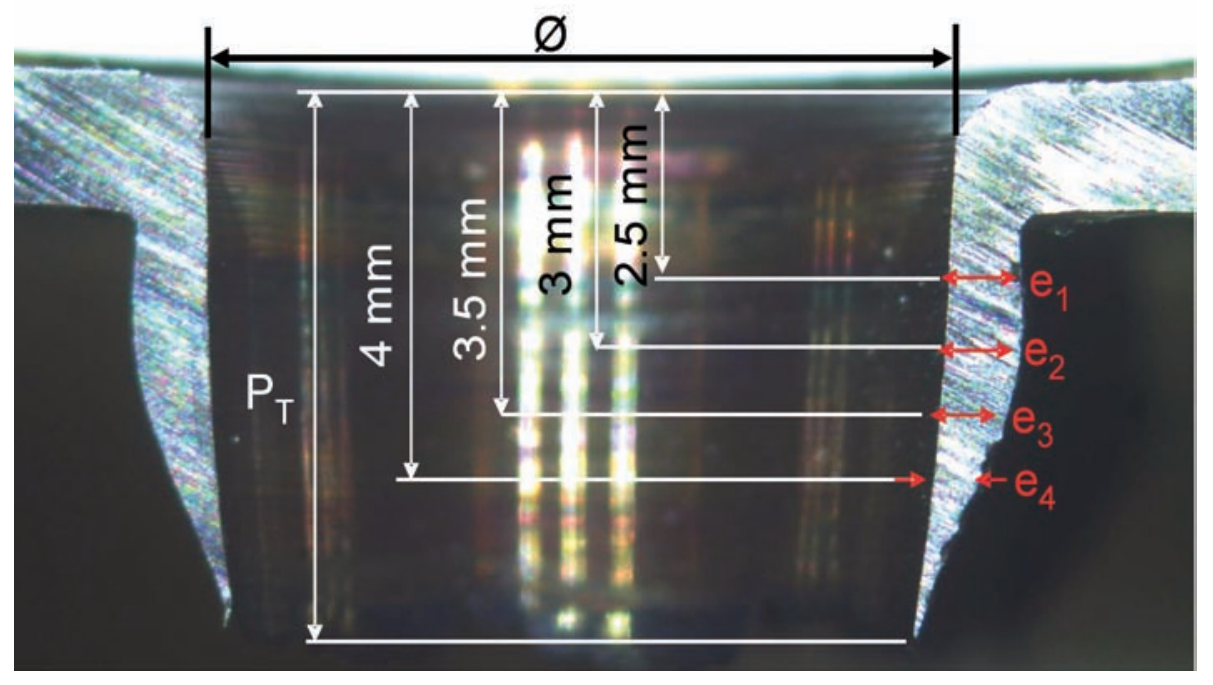

Figura 3. Sección transversal de la rebaba generada.

Figure 3. Cross sections of friction-drilled. 


\section{RESULTADOS Y DISCUSIÓN}

A través de la experimentación se observa un incremento tanto de la fuerza de empuje como del par cuando el espesor de la pieza aumenta. Esto se debe a que el volumen de material a reblandecer y la resistencia a la deformación aumentan con el espesor, lo que conlleva el aumento de las fuerzas de empuje. En cuanto al momento, el área en contacto entre la parte de la rebaba y la herramienta es mayor, generando mayores fuerzas de fricción. Para un espesor de pieza y velocidad de rotación constante, al incrementar el avance, las fuerzas de empuje son mayores. Esto se debe a la disminución del tiempo de contacto entre pieza y herramienta. Se genera menos calor para ablandar el material. Así mismo, cuando se incrementa la velocidad de rotación, manteniendo el avance constate, el valor de las fuerzas de empuje y del par disminuyen. Los mayores valores encontrados de fuerzas y momentos fueron para una combinación del máximo avance con la mínima rotación de ensayo, siendo para la chapa DP 600 un valor de $1780 \mathrm{~N}$ de fuerza de empuje y $5,5 \mathrm{Nm}$ de momento axial. En la chapa DP 800 se obtuvo $2900 \mathrm{~N}$ de fuerza de empuje y $6,8 \mathrm{Nm}$ de momento axial. En la figura 4, se grafican las fuerzas y el momento que se realiza durante el proceso de taladrado.

La temperatura máxima que se alcanza en el proceso es dependiente de la velocidad de rotación, espesor de la chapa y del avance de la herramienta. En la figura 5 de la izquierda, vemos muestras de las temperaturas máximas del proceso en función de la velocidad de giro y del avance de la herramienta en la chapa DP 600 con 1,2 mm de espesor. Se observa que para mayores velocidades de giro, se produce mayor fricción entre herramienta y pieza, lo que origina mayores temperaturas de proceso. La figura 5 de la derecha muestra las temperaturas máximas del proceso en la chapa DP 800 con $1,6 \mathrm{~mm}$ de espesor. Como se ha concluido en la observación de la gráfica anterior, la temperatura del proceso está directamente relacionada con la
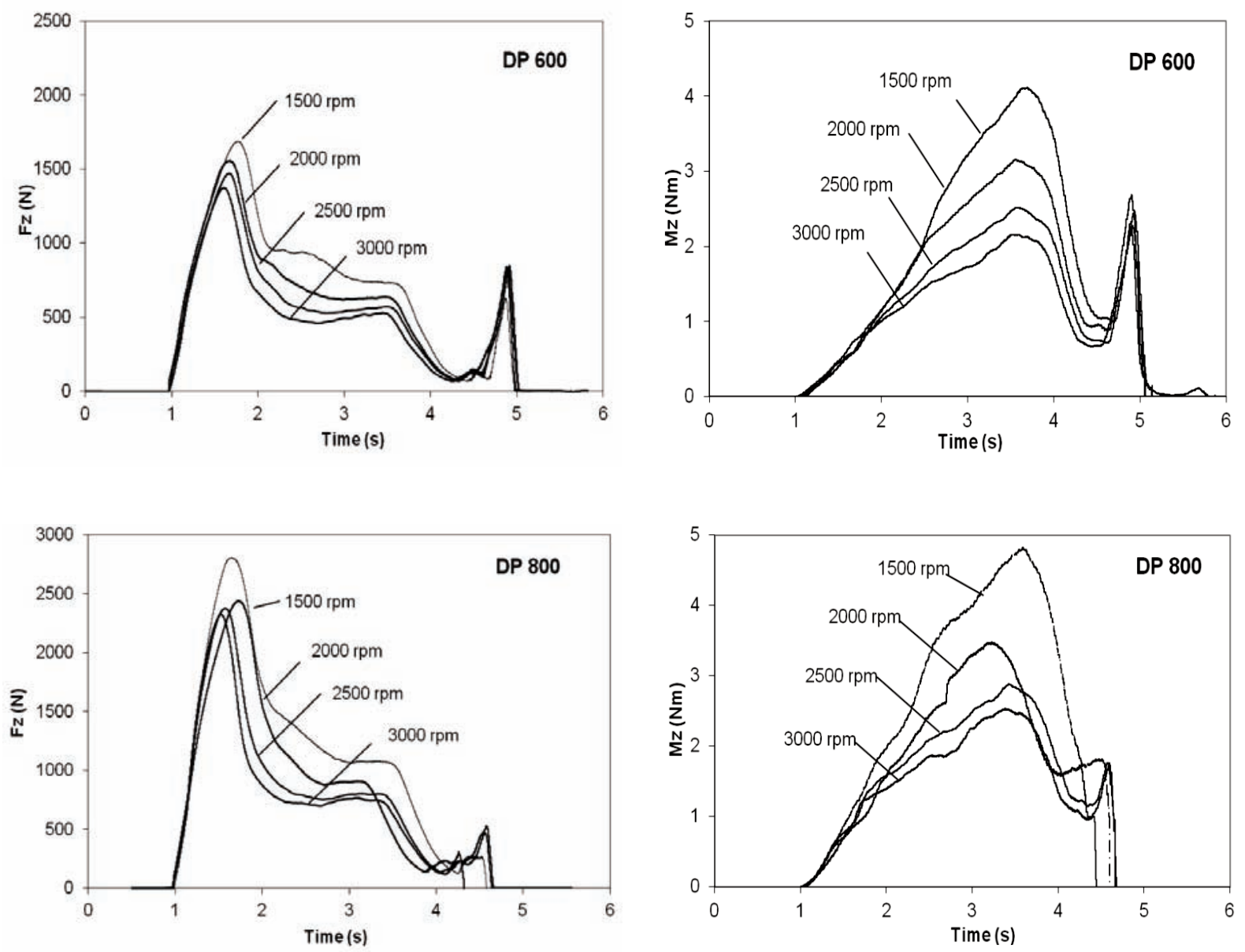

Figura 4. Momentos y fuerzas en acero de doble fase (avance $230 \mathrm{~mm} / \mathrm{min}$ ).

Figure 4. Torque and Thrust force of dual phase steel (feed rate $230 \mathrm{~mm} / \mathrm{min}$ ). 

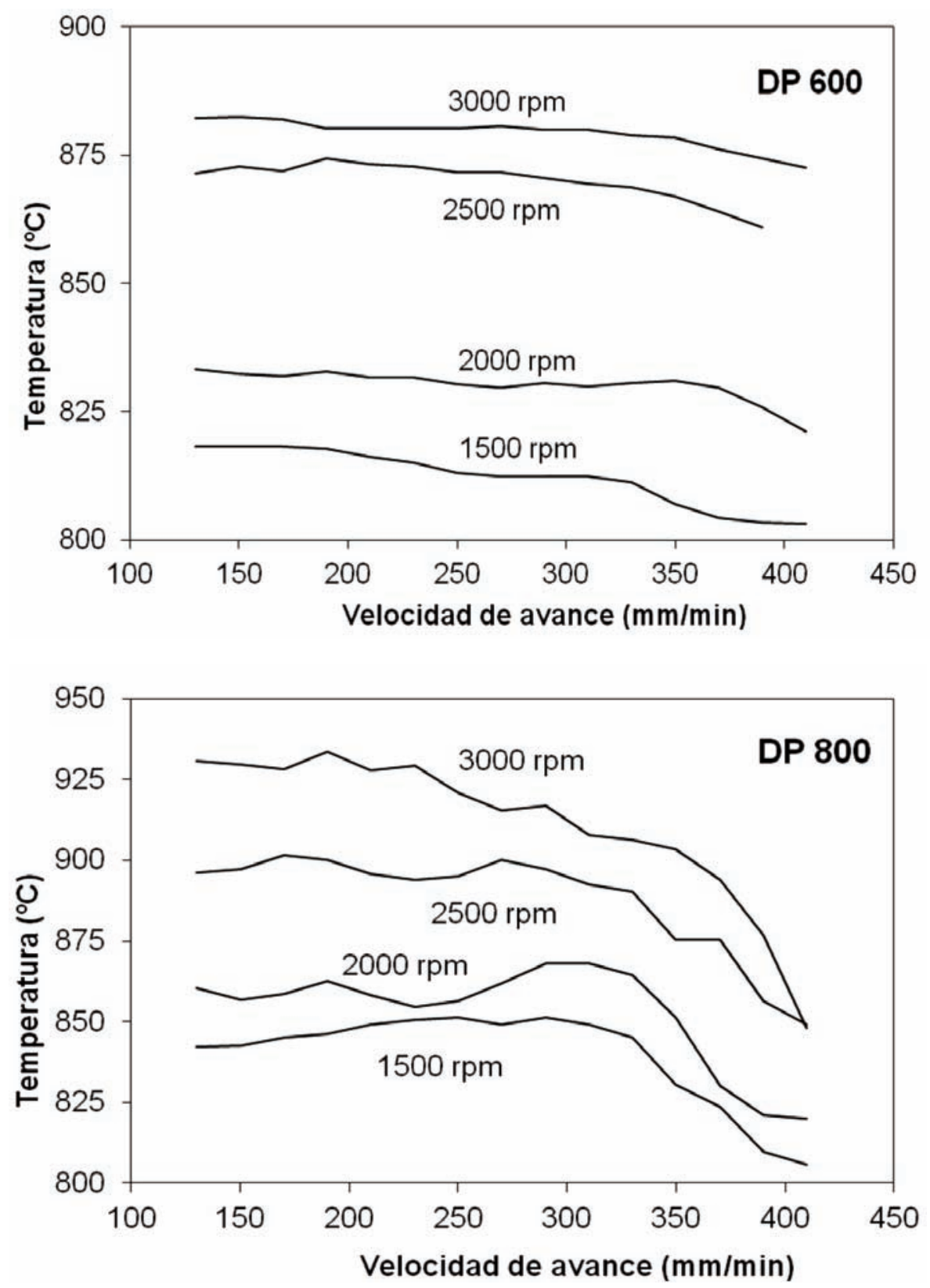

Figura 5. Influencia de los parámetros de taladrado en la temperatura del proceso.

Figure 5. Influence of drilling conditions on the temperature.

velocidad de giro. En cuanto al avance, se observa que un aumento de éste conlleva una disminución de la temperatura máxima, ya que el tiempo de contacto entre la herramienta y la pieza disminuye. Se observa también que utilizando los mismos parámetros durante el proceso y distinto espesor de chapa la temperatura resultante es mayor cuanto mayor el espesor.

En cuanto a la microestructura, en la figura $6 \mathrm{~b}$ ) se muestra las microestructuras dúplex, esto es, de ferrita y martensita, típicas de estos aceros del mate- rial base. La fase clara es la ferrita y la oscura la martensita. El porcentaje de ambas fases depende tanto del contenido de carbono del acero como de la temperatura alcanzada en el proceso. Así, la fracción volumétrica de martensita aumenta conforme crecen el contenido en carbono y la temperatura de recocido. No obstante, los resultados de dureza en el borde del agujero también están influenciados por el contenido en otros elementos de aleación y por los dos espesores utilizados. Debido a la presencia de estos gradientes, en la misma rebaba vamos 
a tener diferentes zonas afectadas por el calor (ZAC). Las transformaciones estructurales del acero de Doble Fase son producidas principalmente en la zona del borde del agujero como vemos en la figura 6 a), a 0,2 $\mathrm{mm}$ desde el borde del agujero. En todos los casos se obtuvieron las microestructuras ferrítico-martensíticas, con la martensita homogéneamente distribuida. Asimismo, los aceros presentan un tamaño de grano menor en el borde del agujero, aumentando el tamaño de grano hasta la estructura original, presentando esta una micro estructura de base ferritica con presencia de martensita formando bandas (la fase oscura), tanto longitudinales de mayor espesor y longitud, como transversales.

Tras el proceso de taladrado, los agujeros suelen roscarse por laminación, por eso motivo, cuanto mayores las dimensiones de las rebabas mejor las resistencias mecánicas finales del agujero roscado. Sin embargo, la calidad de las rebabas también está afectada por la zona de calentamiento. Aunque la variación de la dureza con el espesor no es muy pronunciada, observase una mayor dureza en la zona del borde, lo que responde al tratamiento termomecánico sufrido por las chapas, dado que las regiones más superficiales se enfrían más rápidamente.

De acuerdo con los puntos de medición de microdurezas en la figura 7 , se observa un incremento progresivo de la misma con el contenido en carbono del acero. Los mayores valores de microdurezas encontradas en el caso de DP 600 fueron con las mayores temperaturas, o sea, la combinación del menor avance y mayor rotación, como vemos en la figura 9. Esto se debe por el mayor tiempo de contacto durante el proceso entre la herramienta y la chapa consecuentemente un aumento de temperatura, recristalización y aumento de dureza. Sin embargo en la chapa DP 800 donde las temperaturas de ensayos fueron mayores en comparación con la chapa DP 600, los datos de microdurezas en el borde del agujero fueron similares en comparación a la microdureza de su material base, pues la martensita original del acero DP se reviene por el ciclo térmico del proceso, produciendo un ablandamiento y posterior recuperación en dicha zona respecto de los valores de microdurezas obtenidos para la condición original.

La medición del diámetro y los espesores de los agujeros se realizaron con el fin de analizar las tolerancias obtenidas del agujero en el proceso, en función de diferentes parámetros. En la figura 8 se recoge la dimensión del diámetro del agujero en función de la velocidad de avance y velocidades de giro de la herramienta. Se observa una tendencia que para un mismo espesor de chapa, el incremento en la velocidad de giro provoca un aumento en el diámetro del agujero. El incremento de la velocidad de avance, manteniendo constante la velocidad de giro, resulta en agujeros con diámetros menores.

En la figura 10 se grafica la profundidad de la rebaba $(P t)$ y los espesores (e) en función de la velocidad de giro de la herramienta y de la velocidad de avance empleada en el acero DP 800 de 1,6 $\mathrm{mm}$ de espesor. Se observa que la profundidad de la rebaba está relacionada directamente con el espesor inicial de la pieza. Para un mismo espesor
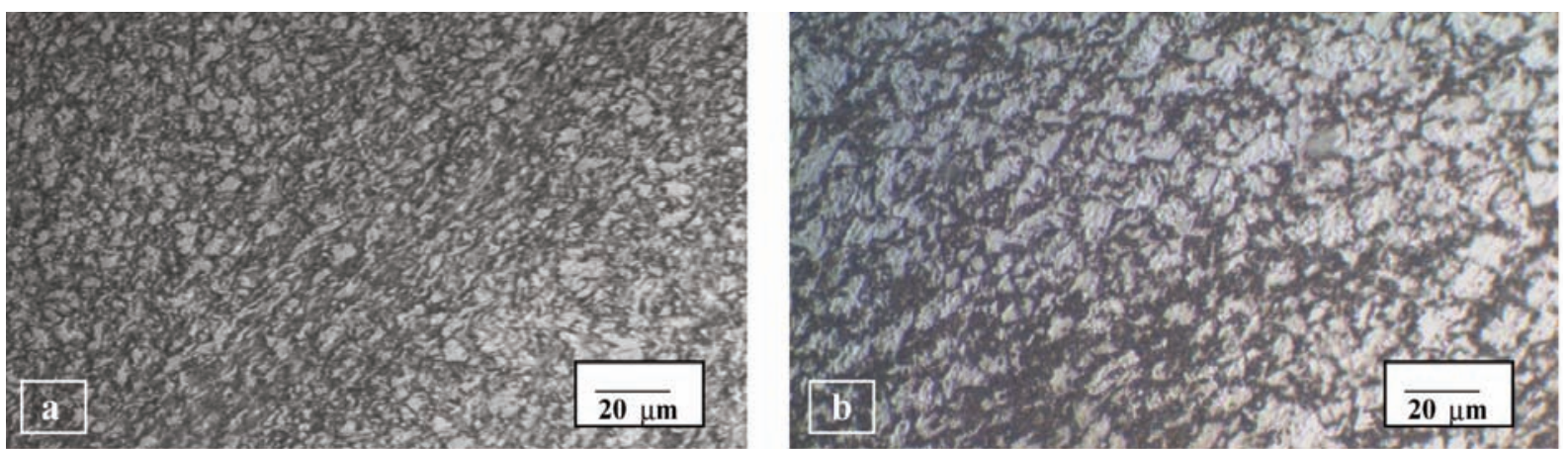

Figura 6. Microestructura del acero Doble Fase DP800 atacada con Nital 2 \% (1000x). La observación microscópica permite apreciar: a) La estructura ferrífico martensítica afectada térmicamente en el borde del agujero. b) La estructura del material base, la fase clara es la ferrita y la oscura la martensita.

Figure 6. Dual Phase DP800 microstructure after etching with $2 \%$ Nital (1000x). The observation shows: a) Microstructure ferrite/martensite with heat-affected zone near the hole edge. b) Base material, martensite islands (dark) in ferrite matrix (bright). 


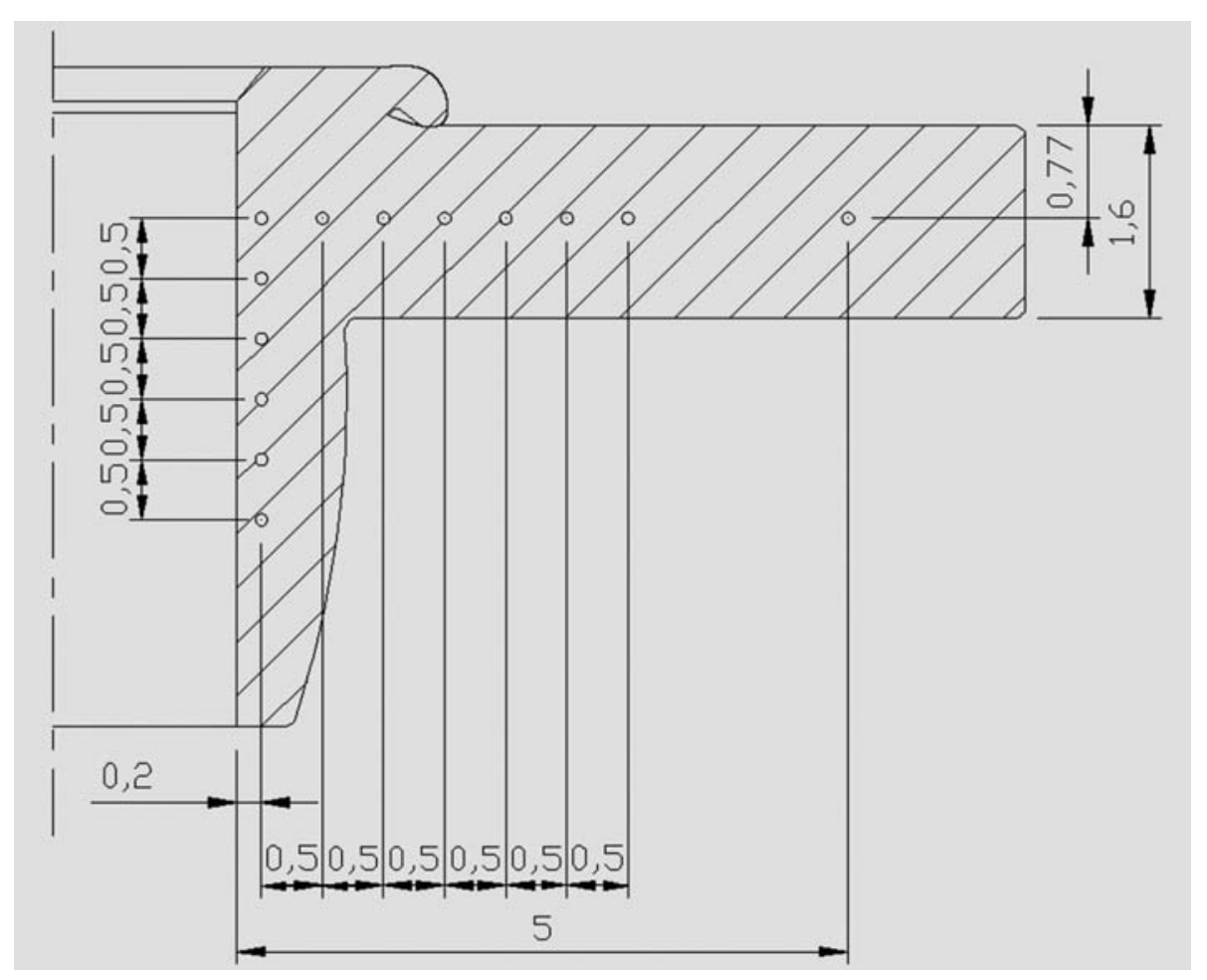

Figura 7. Puntos de medición de microdurezas y metalografias.

Figure 7. Metallographic observation positions and hardness test points.

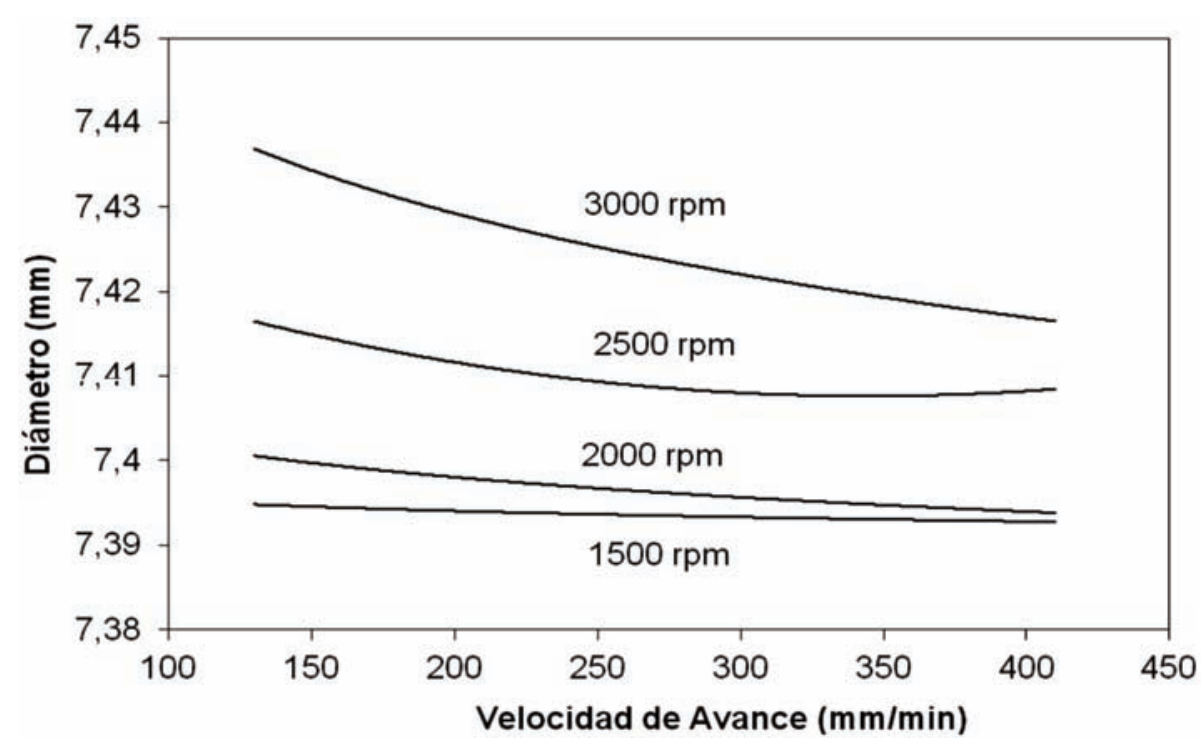

Figura 8. Influencia de los parámetros de taladrado en el diámetro del agujero.

Figure 8. Influence of drilling conditions on the hole diameter.

de pieza, el aumento en la velocidad de giro provoca un incremento del calor generado en el proceso. Esto contribuye a la generación de rebabas de mayores profundidades. El incremento de la velocidad de avance, manteniendo constante la velocidad de giro, genera rebabas de menor pro- 

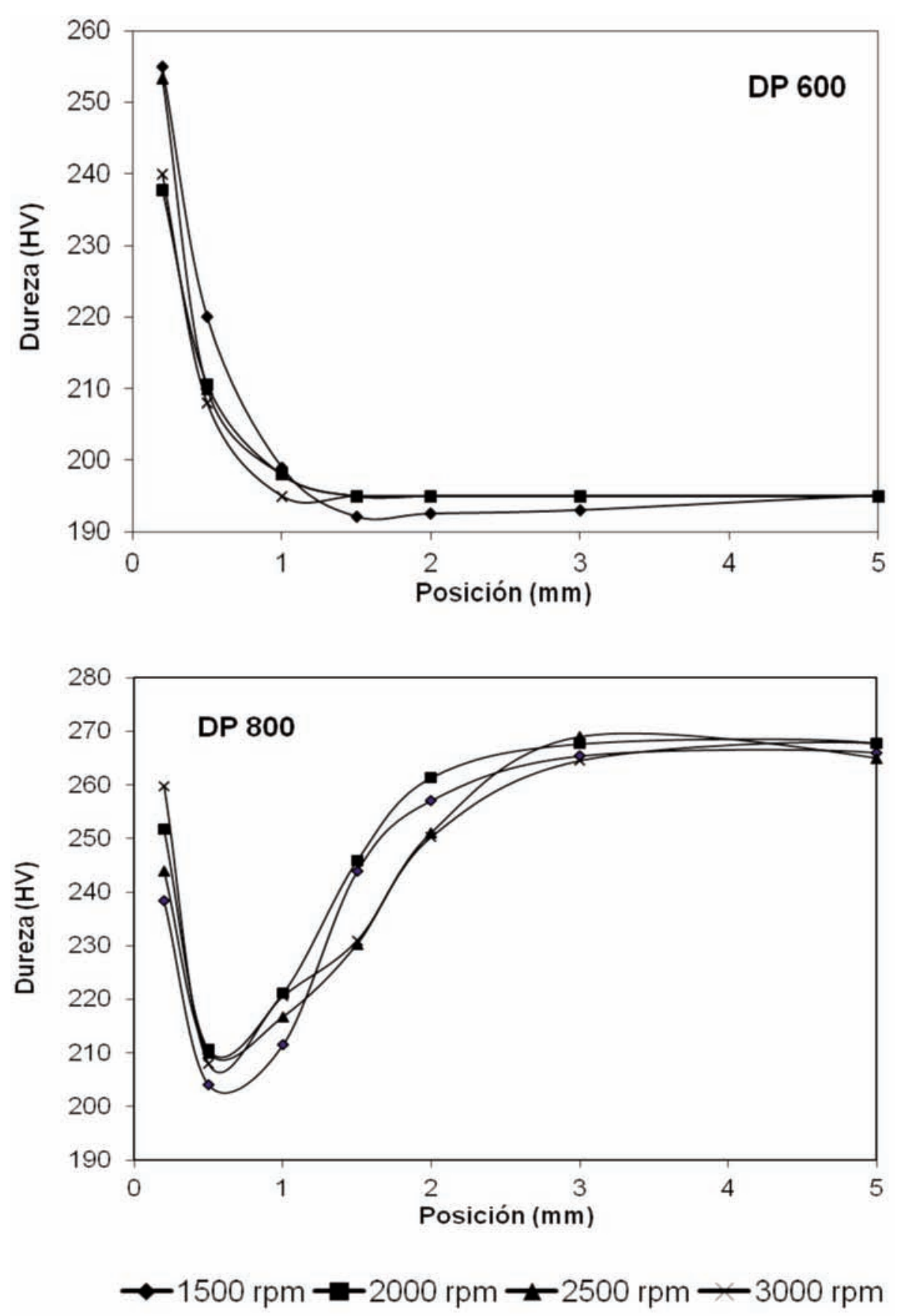

Figura 9. Valores de microdurezas en diferentes posiciones y velocidades de giro (avance $130 \mathrm{~mm} / \mathrm{min}$ ).

Figure 9. Hardness of different positions from hole edge under different drilling speeds (feed rate $130 \mathrm{~mm} / \mathrm{min}$ ).

fundidad, ya que el tiempo de contacto entre la herramienta y el material es menor, se genera menos calor y la facilidad para que el material fluya disminuye. En las mediciones realizadas se observa que el espesor $e_{1}$ y $e_{2}$ tienen un valor similar aunque $e_{2}$ es ligeramente superior. Seguidamente el espesor $e_{3}$ es inferior a $e_{1}$. Se observa la influencia de los parámetros del proceso en los espesores medidos.
Los espesores $e_{1}$ y $e_{2}$ son mayores para velocidades de rotación bajas. Los espesores $e_{3}$ y $e_{4}$ son mayores para velocidades de giro altas, al aumentar el calor generado en el proceso facilita la deformación del material. En este caso los espesores están influenciados por la velocidad de avance, a medida que aumenta la velocidad de avance los espesores medidos son mayores. 

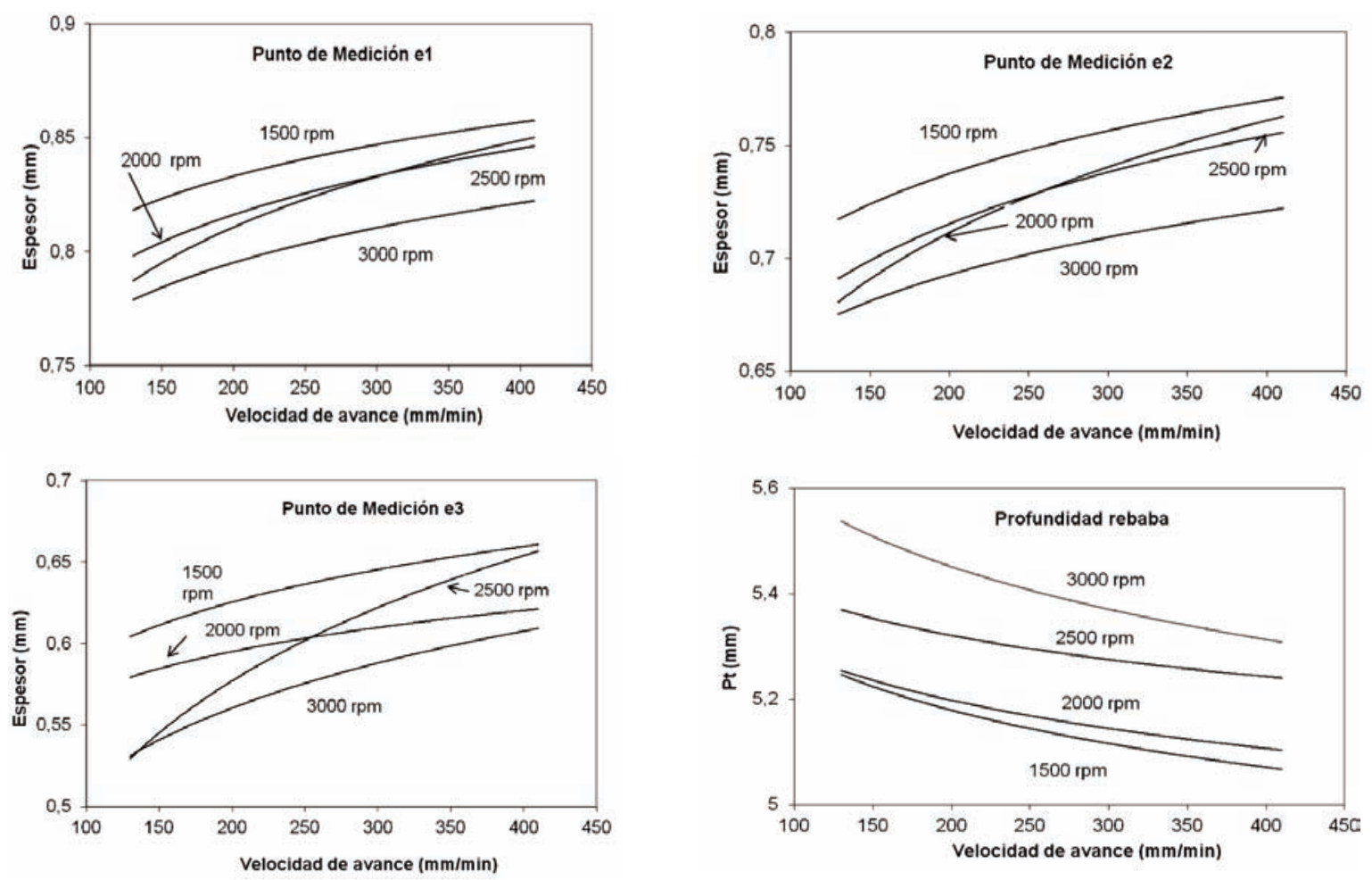

Figura 10. Influencias de los parámetros de taladrado en la profundidad de rebaba.

Figure 10. Influence of drilling conditions in burr depth.

\section{CONCLUSIONES}

- El taladrado por fricción es una técnica rápida, económica y de fácil aplicación para la realización de agujeros en chapas de acero Doble Fase. A través de la experimentación se observa un incremento tanto de la fuerza de empuje como del par cuando el espesor de la pieza aumenta. Esto se debe a que el volumen de material a reblandecer y la resistencia a la deformación aumentan con el espesor. En cuanto al momento axial, el área en contacto entre la parte de la rebaba y la herramienta es mayor, generando mayores fuerzas de fricción. Además, para un espesor de pieza y velocidad de rotación constate, al incrementar el avance, las fuerzas de empuje (axiales) son mayores.

- En el taladrado por fricción el material sufre calentamiento al mismo tiempo que deformación y es sometido a diferentes tasas de deformación y temperatura. Debido a la presencia de estos gradientes, en la misma rebaba se tiene diferentes microestructuras. Los mayores valores de microdurezas encontradas fueron con las mayores temperaturas, o sea, la combinación del menor avance y mayor rotación.

- La profundidad total de la rebaba generada también depende del espesor de partida. A mayor espesor, mayor es el volumen de material a deformar, por lo que se obtienen mayores rebabas. Por otro lado, la profundidad total de la rebaba también está condicionada por los parámetros del proceso. El material tiene más capacidad para fluir si el calor generado como consecuencia de la fricción entre la pieza y la herramienta es mayor. Esto se refleja en las mediciones de temperatura realizadas. Para velocidades de avance lentas, el tiempo en contacto entre material y herramienta es mayor, por lo que la temperatura alcanzada en el proceso es superior a la temperatura obtenida cuando el avance es máximo. La velocidad de rotación de la herramienta también influye en la temperatura alcanzada, cuanto mayor es la velocidad de rotación, mayor es la temperatura experimentada. Así pues, la máxima profundidad de la rebaba se obtiene empleando la máxima velocidad de rotación y la mínima velocidad de avance de las definidas en los ensayos.

\section{Agradecimientos}

Se agradece el apoyo recibido por los compañeros Asier Fernández, el Maestro Eduardo Sasia y en general alumnos y doctorandos del taller del Dept. de 
Ingeniería Mecánica de la Universidad del País Vasco UPV/EHU.

\section{REFERENCIAS}

[1] J.L Bocos, F. Zubiri, F. Garciandía, J. Peña, A. Cortiella, J. M. Berrueta y F. Zapiráin, Rev. Metal. Madrid 40 (2004) 146-151.

[2] W.L. Weingaertner, F. Tikal y J.C. Lopes, Int. J. Manuf. Sci. Prod. 1 (1997) 41-50.

[3] S.F. Miller, R. Li, H. Wang, y A.J. Shih, Int. J. Manuf. Sci. Eng. 128 (2006) 802-810.

[4] J. Qu y P.J. Blau, Int. J. Manuf. Sci. Eng. (2008) 130.

[5] S.F. Miller, J. Tao y A.J. Shih, Int. J. Mach. Tool. Manu. 46 (2006) 1.526-1.535.

[6] S.F. Miller, P.J. Blau y A.J. Shih, Int. J. Mach Tool. Manu. 47 (2007) 1.636-1.645.
[7] S.M. Lee, H. Ming-Cho, F. Yuan-Huang y B. Hwa-Yan, Int. J. Mach. Tool. Manu. 49 (2009) 81-88.

[8] S.M. Lee, H. Ming-Cho y L. Dai-Yang, J. Mater. Process. Tech. 207 (2008) 81-88.

[9] W.L. Weingaertner, F. Tikal y J.C. Lopes, Int. Manuf. Eng. Conference, Connecticut, EE.UU., 1996, pp. 227-231.

[10] A. J. Hoogenboom, EE.UU., Patent № 4454 741 (1984).

[11] G.D. Head, W.C. Le Master, L.P. Bredesky, and D.C. Winter, EE.UU, Patent № 4428214 (1984).

[12] M. Eckhardt, Tesis Doctoral, Facultad de Ingeniería Mecánica, Universidad Federal de Santa Catarina, 2003.

[13] S.F. Miller, Tesis Doctoral, Facultad de Ingeniería Mecánica, Michigan University, 2006. 